Aspirin. In a descending order, aPL, multifetal gestation, lupus nephritis and nulliparity were associated with a higher LDA use, whereas the other risk factors were not.

Preconception counselling significantly increased Aspirin administration [OR 2.36 (95\%-CI: 1.23-4.63), p<0.01], especially in those at high risk [OR 4.12 (95\%-CI 1.72-10.16), $\mathrm{p}<0.001]$. Overall, LDA use increased from 1995 to $2019\left(\chi^{2}\right.$ test for trend in proportions, $\mathrm{p}<0.001)$.

Conclusions We found a high prevalence of pre-eclampsia risk factors and infrequent use of aspirin in pregnant SLE patients. Preconception counselling increases the LDA use and thereby can improve pregnancy outcomes.

\section{P77 EVALUATION OF DISEASE ACTIVITY AT CONCEPTION IN A PROSPECTIVE COHORT OF SLE PREGNANCIES}

Maddalena Larosa, Vanessa Ochrim, Anna Ghirardello, Margherita Zen, Mariele Gatto, Luca laccarino, Andrea Doria. Dept. of Rheumatology, University of Padova, Padova, Italy

\subsection{6/lupus-2020-eurolupus. 122}

Background/Purpose Systemic lupus erythematosus (SLE) is an autoimmune disease, mostly affecting women during their childbearing age. Disease remission before conception is pivotal for a favorable pregnancy outcome. The aims of our study were: 1)to evaluate maternal and fetal complications in a cohort of pregnant SLE patients; 2)to compare Systemic Lupus Erythematosus Disease Activity Score (SLE-DAS) and Systemic Lupus Erythematosus Disease Activity Index 2000 (SLEDAI$2 \mathrm{~K})$ in the 6 months before conception as predictors of maternal/fetal complications.

Methods All SLE pregnancies were prospectively included until August 2019 at our Unit (Rheumatology Department, University of Padova). All women were monthly followed throughout gestation and at least in one occasion in the 6 months before conception. Disease activity was assessed before pregnancy by SLEDAI-2k and SLEDAS. During gestation, we evaluated SLE activity by the Systemic Lupus Erythematosus Pregnancy Disease Activity Index (SLEPDAI) and the SLEDAS index adapted to pregnancy (SLEPDAS). At conception, we considered 'active':1)patients with a SLEDAS $>2.08$; 2)clinically active patients according SLEDAI-2k treated with $\geq 5 \mathrm{mg}$ /day of prednisone (immunosuppressants and antimalarials were allowed). Statistics were performed with SPSS (V.24.0).

Results We enrolled 115 pregnancies in 76 patients (table 1). We found 29 (25.2\%) pregnancies with at least one maternal complication and 39 (33.9\%) with at least 1 fetal adverse outcome. Positive anti-dsDNA and active disease according SLEDAS (SLEDAS $>2.08$ ) at conception resulted to be predictors of maternal complications $(\mathrm{p}=0.036, \mathrm{OR}=2.71, \mathrm{CI} 95 \%$ 1.07-6.87; $\mathrm{p}=0.008, \mathrm{OR}=4.46$, CI 95\% 1.48-13.49, respectively). Conversely, active disease according SLEDAI- $2 \mathrm{k}$ did not result predictive of maternal complications $(p=0.255$, $\mathrm{OR}=2.16$, CI $95 \%$ 9.57-8.16). No predictors of fetal complications were observed.

Conclusions SLE-DAS $>2.08$ and positive anti-dsDNA are risk factors of maternal complications while active SLE according to SLEDAI-2k does not seem to be a predictor of such complications.
Abstract P77 Table 1 Demographic, clinical and serologic features of 115 SLE pregnancies

\begin{tabular}{|c|c|}
\hline Age at conception, yrs, mean \pm SD & $33.3 \pm 4.6$ \\
\hline Disease duration at conception, yrs, mean \pm SD & $11.0 \pm 0.6$ \\
\hline \multicolumn{2}{|l|}{ Previous organ involvement, $\mathrm{N}(\%)$} \\
\hline articular, N (\%) & $87(75.7)$ \\
\hline cutaneous, N (\%) & $74(64.3)$ \\
\hline hematological, N (\%) & $37(32.2)$ \\
\hline NPSLE, N (\%) & $11(9.6)$ \\
\hline renal, N (\%) & $45(39.1)$ \\
\hline serositis, N (\%) & $10(8.7)$ \\
\hline systemic, N (\%) & $43(37.4)$ \\
\hline vascultiis, $\mathrm{N}(\%)$ & $3(2.6)$ \\
\hline sicca syndrome, N (\%) & $10(8.70)$ \\
\hline \multicolumn{2}{|l|}{ SLE activity within 6 months before conception } \\
\hline Active disease at conception according SLEDAI-2k, N (\%) & $15(13.0)$ \\
\hline Active disease at conception according SLEDAS, $\mathrm{N}(\%)$ & $17(14.8)$ \\
\hline SLEDAI-2k witin 6 months before conception, median (IQR) & $2.0(2.0 ; 4.0)$ \\
\hline SLEDAS witin 6 months before conception, median (IQR) & $1.3(1.1 ; 2.1)$ \\
\hline \multicolumn{2}{|l|}{ Delivery } \\
\hline Live births, N (\%) & $99(86.1)$ \\
\hline Week of delivery, median (IQR) & $38(36.7 ; 39.0)$ \\
\hline \multicolumn{2}{|l|}{ Antibodies profile at conception, $\mathrm{N}(\%)$} \\
\hline anti-dsDNA, N (\%) & $56(49.1)$ \\
\hline anti-RNP, N (\%) & $20(17.4)$ \\
\hline anti-Sm, N (\%) & $13(11.3)$ \\
\hline anti-SSA, N (\%) & $38(33.0)$ \\
\hline anti-SSB, N (\%) & $13(11.3)$ \\
\hline LAC, N (\%) & $12(10.4)$ \\
\hline 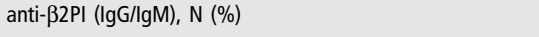 & $12(10.4)$ \\
\hline anti-aCL (IgG/lgM), N (\%) & $14(12.2)$ \\
\hline triple aPL positivity, N (\%) & $3(2.6)$ \\
\hline
\end{tabular}

Legend to Table 1: SLE: systemic lupus erythematosus; NPSLE: neuropsychiatric SLE; SLEDAI-2k: Systemic Lupus Erythematosus Disease Activity Index 2000; SLEDAS: Systemic Lupus Erythematosus Disease Activity Score; SLEPDAI: Systemic Lupus Erythematosus Pregnancy Disease Activity Index; SLEPDAS: Systemic Lupus Erythematosus Pregnancy Disease Activity Score; anti-dsDNA: anti-double stranded DNA; LAC: lupus anticoagulant; anti- $\beta 2 \mathrm{PI}$ : anti- beta-2-glicoprotein; anti-aCL: anti-cardiolipin; aPL: anti-phospholipid antibodies.

\section{P78 OUTCOMES OF SYSTEMIC LUPUS ERYTHEMATOSUS PREGNANCIES AND ASSOCIATED FACTORS IN SUB- SAHARAN AFRICA: A SYSTEMATIC SCOPING REVIEW}

${ }^{1}$ Mickael Essouma, ${ }^{1} J a n$ René Nkeck, ${ }^{2,3}$ Jean Joel Bigna, ${ }^{4}$ Grâce Anita Nkoro, ${ }^{5}$ Stéphane Ralandison, ${ }^{6}$ Eric Hachulla. ${ }^{1}$ Faculty of Medicine and Biomedical Sciences, University of Yaoundé I, Yaoundé; ${ }^{2}$ Centre Pasteur of Cameroon, Yaoundé, Cameroon; ${ }^{3}$ Faculty of Medicine, University of Paris Sud XI, Le Kremlin-Bicêtre, France; ${ }^{4}$ Yaoundé Gyneco-Obstetric and Pediatric Hospital, Yaoundé, Cameroon; ${ }^{5}$ Faculty of Medicine of Toamasina, Tamatave, Madagascar, ${ }^{6}$ Claude Huriez Hospital, Lille University, Lille, France

\subsection{6/lupus-2020-eurolupus. 123}

Background We have previously observed increased morbidity and mortality in Native sub-Saharan Africans with systemic lupus erythematosus (SLE). Herein, we report a survey of the literature on outcomes of SLE pregnancies and associated factors in sub-Saharan Africa.

Methods We searched PUBMED, EMBASE and African Journals Online to identify relevant studies published between January 1, 2000 and October 28, 2019. We then conducted a qualitative synthesis. 
Results Four studies were included, out of 2693 records. One hundred and thirty-seven pregnancies were recorded in 102 women with mean age ranging from 27.2 years to 39.9 years in 1992-2018. At conception, the predominant organ manifestations were renal and cutaneous, whereas disease activity measures were generally unavailable. Livebirths occurred in $57 \%-82 \%$ of pregnancies and pregnancy loss in $18 \%-43 \%$. Other major adverse pregnancy outcomes (figure 1) were: SLE flare mainly presented as nephritis, pre-eclampsia (9\%-33\%), pre-term birth (up to $56 \%$ of livebirths), and low birthweight (14\%-85\% of live births). No study clearly reported risk factors for adverse pregnancy outcomes. Rather, the factors likely associated with adverse obstetric, fetal and neonatal outcomes included SLE flare (especially nephritis), maternal serological status during pregnancy and hypertensive disorders of pregnancy.

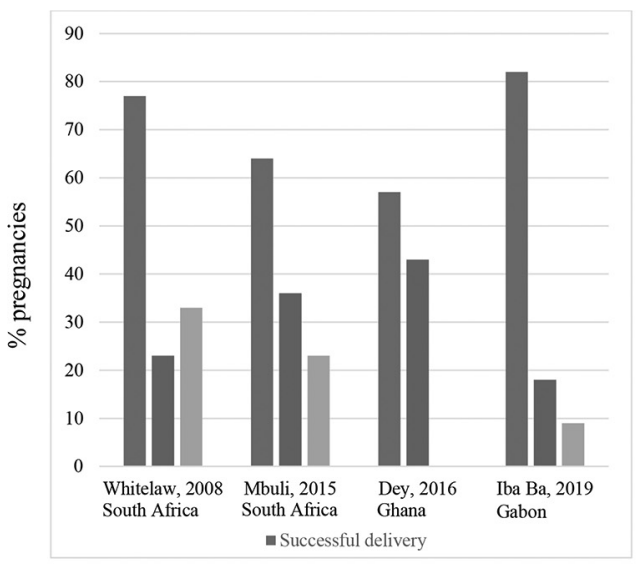

A. Obstetric complications

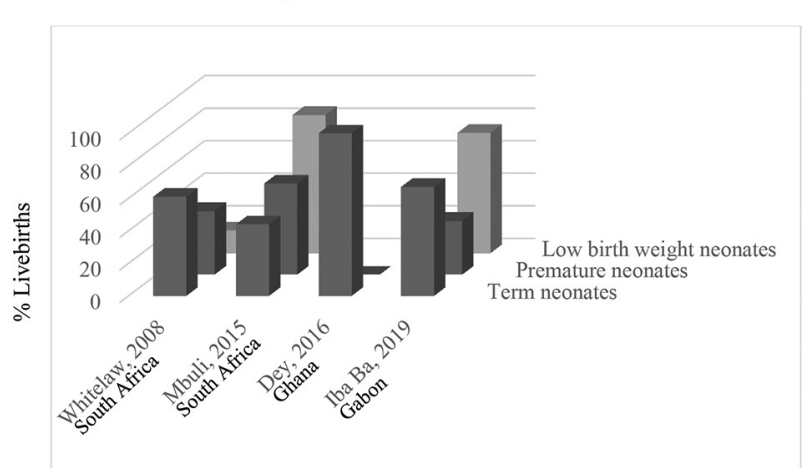

B. Neonatal complications

Abstract P78 Figure 1 Main complications of systemic lupus erythematosus pregnancies in sub-Saharan Africa in 1992-2018.

Conclusions Contemporary available literature reports overall low rates of livebirth in sub-Saharan African women with SLE. The main adverse pregnancy outcomes reported are : SLE flare, pregnancy loss, pre-eclampsia, pre-term birth and low birthweight. Although no risk factor has been clearly mentioned, SLE flare presented as nephritis is the major factor likely associated with adverse pregnancy outcomes. Thorough investigation is warranted.

Acknowledgements None.

\section{P79 PREDICTORS OF ADVERSE NEONATAL OUTCOME DURING THE PREGNANCY OF WOMEN WITH ANTIPHOSPHOLIPID SYNDROME IN THE FRENCH GR2 PROSPECTIVE STUDY}

${ }^{1}$ Anne Murarasu, ${ }^{1}$ Gaëlle Guettrot-Imbert, ${ }^{1}$ Véronique Le Guern, ${ }^{2}$ Francois Maurier, ${ }^{3}$ Patrick Jego, ${ }^{4}$ Estibaliz Lazaro, ${ }^{5}$ Alban Deroux, ${ }^{6}$ Maëlle Le Besnerais, ${ }^{7}$ Odile SouchaudDebouverie, ${ }^{8}$ Pauline Orquevaux, ${ }^{9}$ Catherine Deneux-Tharaux, ${ }^{1}$ Nathalie CostedoatChalumeau, the GR2 study group. 'Internal Medicine Dept., Hôpital Cochin AP-HP, Paris; ${ }^{2}$ Internal Medicine Dept., Hôpital Belle-Isle, Metz; 'Internal Medicine Dept., CHU de Rennes, Rennes; ${ }^{4}$ Internal Medicine Dept., CHU de Bordeaux, Bordeaux; ${ }^{5}$ Internal Medicine Dept., CHU de Grenoble, La Tronche; ${ }^{6}$ Internal Medicine Dept., CHU de Rouen, Rouen; ${ }^{7}$ Internal Medicine Dept., CHU de la Milétrie, Poitiers; ${ }^{8}$ Internal Medicine Dept., CHU Robert Debré, Reims; ${ }^{9}$ EPOPé Team, University of Paris, Epidemiology and Statistics Research Center/ CRESS, INSERM, INRA F-75004, Paris, France

\subsection{6/lupus-2020-eurolupus.124}

Introduction Data about the predictors of adverse neonatal outcome in pregnant women with antiphospholipid syndrome (APS) are sparse. The main study on this subject is the prospective American study PROMISSE on 144 carriers of antiphospholipid antibodies. ${ }^{1}$ We report the first results of the French study, GR2.

Methods Inclusion criteria were: (1) an ongoing pregnancy at 12 weeks of gestation (WG), (2) conceived before May 2018, (3) of a woman with APS. ${ }^{2}$ Exclusion criteria were proteinuria (ratio $>1 \mathrm{~g} / \mathrm{g}$ ), serum creatinine $>100 \mu \mathrm{mol} / \mathrm{L}$, and multifetal pregnancy.

The composite primary outcome included an intrauterine fetal death (IUFD), a neonatal death, placental insufficiency (fetal growth restriction, preeclampsia, HELLP syndrome, and/ or placental abruption) leading to a delivery $\leq 34 \mathrm{WG}$, and/or a small for gestational age (SGA) $\leq 3$ rd percentile.

Results We analysed 119 pregnancies in 119 patients: 53\% thrombotic and 47\% obstetric only APS; 60\% had lupus anticoagulant (LA) and 26\% associated SLE. Treatment included aspirin (99\%), heparin (98\%, in the therapeutic range for $50 \%)$, and hydroxychloroquine $(62 \%)$.

The primary outcome was observed in $14 \%$ of pregnancies: $3 \%$ IUFD, $8 \%$ delivery $\leq 34 \mathrm{WG}$ due to placental insufficiency and/or 5\% of SGA (and no neonatal death). It was observed in $20 \%$ of the pregnancies with LA compared with $7 \%$ for women negative for LA $(P=0.096)$. This outcome did not differ according to anticardiolipin or antiß2GP1 antibody status, or prior thrombosis (14\%, versus $14 \%$ for women with only obstetric APS). There was a trend toward more adverse neonatal outcomes in case of prior arterial thrombosis $(29 \%$ versus $12 \%, P=0.07)$. The rate of adverse neonatal outcomes did not differ among the women with or without associated SLE (29\% versus $26 \%, P=0.77$ ).

In multivariate analysis, independent features associated with pregnancy failure were: in a serological model, LA positive status $(\mathrm{ORa}=8.5,95 \% \mathrm{CI}[1.0-71.5])$ and in a serological and clinical model, 'non-white' skin colour $(\mathrm{ORa}=6.1,95 \% \mathrm{CI}$ [1.1-34.7]).

Conclusion The rate of adverse neonatal outcomes tended to be lower than in the PROMISSE study (14\% versus 19\%), although the women included here were theoretically more severe (definite APS). One potential explanation might be that our patients were all treated. In keeping with the PROMISSE study, LA and skin colour were the main predictors of neonatal outcome.

Acknowledgement Fondation pour la Recherche Médicale (M2R201806006403) 